\title{
Front Matter: Volume 9163
}

, "Front Matter: Volume 9163," Proc. SPIE 9163, Plasmonics: Metallic Nanostructures and Their Optical Properties XII, 916301 (8 October 2014); doi: $10.1117 / 12.2086162$

SPIE Event: SPIE NanoScience + Engineering, 2014, San Diego, California, United SPIE. States 


\title{
PROCEEDINGS OF SPIE
}

\section{Plasmonics: Metallic Nanostructures and Their Optical Properties XII}

\author{
Allan D. Boardman \\ Editor
}

17-21 August 2014

San Diego, California, United States

Sponsored and Published by

SPIE 
The papers included in this volume were part of the technical conference cited on the cover and title page. Papers were selected and subject to review by the editors and conference program committee. Some conference presentations may not be available for publication. The papers published in these proceedings reflect the work and thoughts of the authors and are published herein as submitted. The publisher is not responsible for the validity of the information or for any outcomes resulting from reliance thereon.

Please use the following format to cite material from this book:

Author(s), "Title of Paper," in Plasmonics: Metallic Nanostructures and Their Optical Properties XII, edited by Allan D. Boardman, Proceedings of SPIE Vol. 9163 (SPIE, Bellingham, WA, 2014) Article CID Number.

ISSN: 0277-786X

ISBN: 9781628411904

Published by

SPIE

P.O. Box 10, Bellingham, Washington 98227-0010 USA

Telephone +1 3606763290 (Pacific Time) · Fax +1 3606471445

SPIE.org

Copyright @ 2014, Society of Photo-Optical Instrumentation Engineers.

Copying of material in this book for internal or personal use, or for the internal or personal use of specific clients, beyond the fair use provisions granted by the U.S. Copyright Law is authorized by SPIE subject to payment of copying fees. The Transactional Reporting Service base fee for this volume is $\$ 18.00$ per article (or portion thereof), which should be paid directly to the Copyright Clearance Center (CCC), 222 Rosewood Drive, Danvers, MA 01923. Payment may also be made electronically through CCC Online at copyright.com. Other copying for republication, resale, advertising or promotion, or any form of systematic or multiple reproduction of any material in this book is prohibited except with permission in writing from the publisher. The CCC fee code is 0277-786X/14/\$18.00.

Printed in the United States of America.

Publication of record for individual papers is online in the SPIE Digital Library.

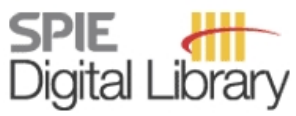

SPIEDigitallibrary.org

Paper Numbering: Proceedings of SPIE follow an e-First publication model, with papers published first online and then in print and on CD-ROM. Papers are published as they are submitted and meet publication criteria. A unique, consistent, permanent citation identifier (CID) number is assigned to each article at the time of the first publication. Utilization of CIDs allows articles to be fully citable as soon as they are published online, and connects the same identifier to all online, print, and electronic versions of the publication. SPIE uses a six-digit CID article numbering system in which:

- The first four digits correspond to the SPIE volume number.

- The last two digits indicate publication order within the volume using a Base 36 numbering

system employing both numerals and letters. These two-number sets start with 00, 01, 02, 03, 04, $05,06,07,08,09,0 A, 0 B \ldots$. 0Z, followed by 10-1Z, 20-2Z, etc.

The CID Number appears on each page of the manuscript. The complete citation is used on the first page, and an abbreviated version on subsequent pages. Numbers in the index correspond to the last two digits of the six-digit CID Number. 


\title{
Contents
}

\author{
vii Authors \\ xi Conference Committee
}

ACTIVE, GAIN, SPASING, AND APPLICATIONS

916305 YHtrium hydride nanoantennas for active plasmonics [9163-4]

$916308 \quad$ Plasmonic black metal polarizers for ultra-short laser pulses [9163-7]

NONLINEARITY, STRINGS, AND MODELS

$91630 \mathrm{~A}$ High accuracy models of sources in FDTD computations for subwavelength photonics design simulations [9163-9]

\section{GRAPHENE PLASMONICS}

9163 OF Strong exciton-plasmon coupling in graphene-semiconductor structures (Invited Paper) [9163-14]

META OUTCOMES

$91630 \mathrm{M}$ Three-dimensional metamaterials: from split ring resonator to toroidal metamolecule (Invited Paper) [9163-22]

\section{PLASMONS, CONTROL, AND TUNABILITY I}

916300 Mode control and loss compensation of propagating surface plasmons (Invited Paper) [9163-25]

$91630 Q \quad$ Surface plasmon mediated inverse polarization transmission through metallic gratings in deep ultraviolet band [9163-27]

$9163 \mathrm{OR} \quad$ Highly tunable ultra-narrow-resonances with optical nano-antenna phased arrays in the infrared [9163-28] 
$91630 \mathrm{~V}$ Comparison of third-order nonlinear optical properties of colloidal gold nanoshells and nanorods [9163-32]

$91630 \mathrm{~W}$ Field enhanced plexitonic coupling between InAs quantum dot and silver film: highly sensitive plasmonic composite [9163-33]

NANOSCALE ACTIVITY I

$91630 Z$ Plasmon-exciton coupling between silver nanowire and two quantum dots (Invited Paper) [9163-35]

$916310 \quad$ Nanoplasmonic hydrogen sensing (Invited Paper) [9163-36]

NANOSCALE ACTIVITY II

916313 Imaging of rectified plasmonic fields on nanoantennas with single nanometer precision [9163-39]

916314 Laser-directed deposition of silver nanostructures [9163-40]

916315 Plasmonic optoelectronics on silicon (Invited Paper) [9163-41]

PLASMONS, CONTROL, AND TUNABILITY II

916317 3D-FEM analysis of SPP excitation through nanoholes in asymmetric metal-insulator-metal structure at tip of circular truncated conical fiber [9163-43]

91631 A Optical nanogap matrices for plasmonic enhancement applications [9163-46]

9163 1B Plasmon-enhanced photothermal response in heterogeneous metallic trimers [9163-47]

ON THE NANOSCALE AGAIN

9163 ID Nano-scale patterns of molybdenum on glass substrate for use in super-resolution imaging with metamaterials [9163-49]

9163 1G Plasmonic nanostructuring through direct laser interference patterning [9163-52]

ABOUT NANOPARTICLES

$916311 \quad$ Numerical prediction of the effect of nanoscale surface roughness on film-coupled nanoparticle plasmon resonances (Invited Paper) [9163-54]

iv 
PLASMONS, NANOSTRUCTURES, AND MODELING

$91631 \mathrm{~K} \quad$ Enhanced up-conversion in nanocrystals coupled to silver nanowires [9163-56]

$91631 \mathrm{M} \quad$ Generation and characterization of plasmonic nanostructures in glass surfaces by means of excimer and solid state laser irradiation [9163-58]

PLASMONS, CONTROL, AND SENSING I

$91631 \mathrm{U} \quad$ Near-field vector analysis of plasmonic structures enhancement on infrared photodetectors [9163-67]

\section{PLASMONS, CONTROL, AND SENSING II}

$91631 \mathrm{~W} \quad$ Polarimetric analysis of the extraordinary optical transmission through subwavelength hole arrays [9163-69]

\section{PATHWAYS TO APPLICATIONS I}

916320 Near-field, back-action cooling and amplification [9163-73]

$916321 \quad$ Polarization manipulation with subwavelength nanoapertures [9163-74]

\section{POSTER SESSION}

$91632 \mathrm{E}$ Optimizing the quantum dot: plasmon interaction in a nano gap waveguide [9163-89]

$91632 \mathrm{~F}$ Transient plasmon-like modes in multi-level quantum emitter systems [9163-90]

$91632 \mathrm{G} \quad$ Tailoring plasmonic field within two-color laser using metallic nanostructure for the generation of high harmonics [9163-91]

$91632 \mathrm{H} \quad$ Optical spectra of noble metal nanoparticles supported on zeolites [9163-92]

$91632 \mathrm{~J} \quad$ Dynamic optical properties of amorphous diamond-like carbon nanocomposite films doped with Cu and Ag nanoparticles [9163-94]

$91632 \mathrm{M} \quad$ Plasmonic influence on the up-conversion luminescence in $\mathrm{NaYF}_{4}: \mathrm{Er}^{3+} / \mathrm{Yb}_{3+}$ nanocrystals [9163-97]

$91632 Q \quad$ Background-suppressed surface-enhanced molecular detection by metamaterial infrared absorber [9163-101]

$91632 R \quad$ Observation of optical domino modes in arrays of non-resonant plasmonic nanoantennas [9163-102] 
916325 The contribution of plasmon-enhanced photoluminescence to the SERS background [9163-103]

$91632 \mathrm{U}$ Coherence changes of partially coherent beams in transmission through subwavelength metallic gratings [9163-105]

$91632 \mathrm{~V}$ Super long-range surface plasmon polaritons in a silver nano-slab waveguide [9163-106]

$91632 \mathrm{~W}$ Optical and structural properties of graphene oxide-noble metal bilayers [9163-107]

$91632 Y \quad$ Plasmonic response of different metals for specific applications [9163-109]

$91632 Z$ High resolution characterization of plasmon resonances in silver nanostructures [9163-110]

916330 Asymmetric gold nanoparticle reduction into polydimethylsiloxane thin films [9163-111]

916331 Increased upconversion quantum yield in plasmonic structures [9163-112]

916332 Geometric optics of gold nanoparticle-polydimethylsiloxane thin films [9163-113]

916334 Optical conductivity of a two-dimensional electron gas with Rashba and Dresselhaus spinorbit coupling [9163-115]

916336 Two-photon activation of o-nitrobenzyl ligands bound to gold surfaces [9163-117]

9163 3B Wafer-scale aluminum nano-plasmonics [9163-122]

$91633 \mathrm{C}$ The field enhancement and optical sensing in the array of almost adjoining metal and dielectric nanorods [9163-123]

$91633 \mathrm{E}$ Exciton-plasmon interaction in core/shell spherical nanoparticles [9163-125]

$91633 \mathrm{H}$ Optical properties of magnetic-plasmonic nanoparticle multilayers [9163-128]

9163 3J An ultra-broadband nano-plasmonic reflector with an asymmetric nano-ring resonator [9163-131]

$91633 \mathrm{~L} \quad$ Focusing surface plasmons by a plasmonic lens [9163-133]

$91633 \mathrm{M}$ Dark and bright modes manipulation for plasmon-triggered photonic devices [9163-134]

916330 Polarizability extraction for rapid computation of Fano resonance in nanoring lattices [9163-136]

$91633 \mathrm{Q}$ Computational electromagnetic analysis of plasmonic effects in interdigital photodetectors [9163-139]

$91633 R \quad$ Kerr effect from single gold nanoparticles [9163-140] 


\section{Authors}

Numbers in the index correspond to the last two digits of the six-digit citation identifier (CID) article numbering system used in Proceedings of SPIE. The first four digits reflect the volume number. Base 36 numbering is employed for the last two digits and indicates the order of articles within the volume. Numbers start with 00, 01, 02, 03, 04, 05, 06, 07, 08, 09, OA, OB...0Z, followed by 10-1Z, 20-2Z, etc.

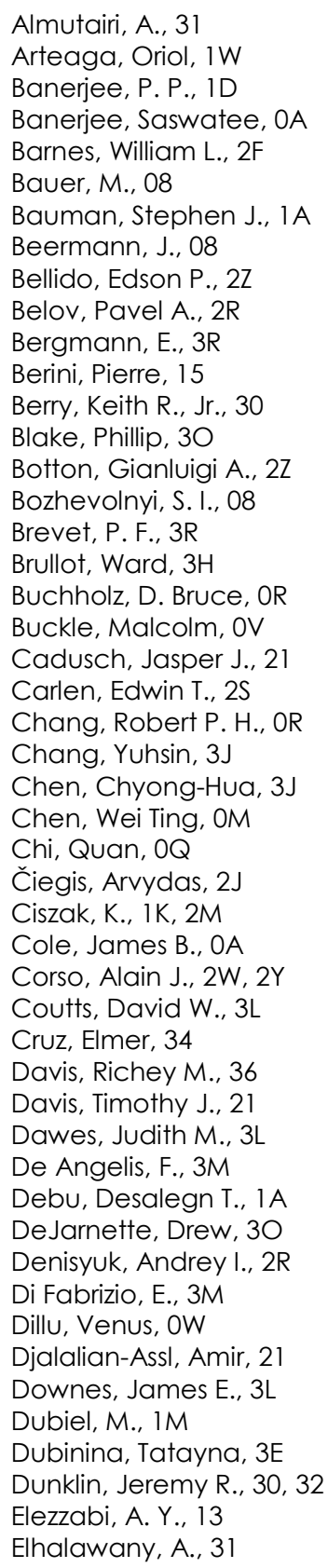

Eriksen, R. L., 08

Fathipour, Vala, 20

Fedotov, Vassili A., OM

Firby, C. J., 13

Forcherio, Gregory T., 30, 32, 30

Frasier, James, 3B

Gardner, Eric, 3B

Gentile, Martin J., 2F

George, Matthew C., 3B

Gerlin, Francesca, 2W, $2 Y$

Giessen, Harald, 05

Gordel, Marta, OV

Griessen, Ronald, 05

Gu, Guiru, 14

Guo, Peijun, OR

Guo, Xi, 36

Guzhva, Mikhail E., 2R

Hassani Nia, Iman, 20

Hayenga, W. E., 31

He, S., 31

Heinz, M., $1 M$

Herzog, Joseph B., 1A, 3Q

Higuchi, Mai, 2V

Hill, Avery M., 1A, 3Q

Holmgaard, T., 08

Hsu, Wei-Lun, OM

$\mathrm{Hu}, \mathrm{J} ., \mathrm{DD}$

Huang, Yao-Wei, OM

Ihlemann, J., $1 \mathrm{M}$

Ishikawa, Atsushi, 2Q

Ivanov, Andrey V., 3C

James, Timothy D., 21

Jia, Zhili, 00

Jiang, Ke, 14

Johnson, N. J. J., 31

Jonin, Ch., 3R

Kahr, Bart, IW

Kang, Guoguo, OQ

Ketterson, John B., OR

Khajavikhan, M., 31

Kik, Pieter G., 1B, 11

Kolkowski, Radoslaw, OV

Krasovskii, Vitaly, 3E

Kreibig, Uwe, 05

Krichevsky, Denis, 3E

Lagarkov, Andrey N., 3C

Lajunen, Hanna, $2 \mathrm{U}$

Langhammer, Christoph, 10

Lantigua, C., 31 
Le Thi Ngoc, Loan, 25

Leissner, T., 08

Lemke, C., 08

Li, H., 1D

Li, Qiang, $0 Z$

Li, Qian-Guang, 2G

Li, Shi-Qiang, OR

Liao, Chun Yen, OM

Liberale, C., 3M

Lin, Xue-Chun, $2 G$

Lin, Yuanhai, $1 G$

López-Bastidas, Catalina, 2H, 34

Lu, Xuejun, $1 \mathrm{U}$

Lumdee, Chatdanai, 11

Machorro, Roberto, $2 \mathrm{H}$

Maćkowski, S., 1K, 2M

Maggini, Michele, $2 \mathrm{~W}$

Magill, Brenden A., 36

Maleki, Alireza, 3L

Manasreh, Omar M., 3Q

Maoz, Ben, IW

Markovich, Gil, IW

Matczyszyn, Katarzyna, OV

Maurice, A., 3R

Maytorena, Jesús A., 34

Meinertz, J., 1M

Meškinis, Šarūnas, 2J

Miyata, Masashi, 2V

Mohseni, Hooman, 20

Mukhin, Ivan S., 2R

Murai, K., 17

Nakano, M., 17

Nardello, Marco, 2W

Natelson, Douglas, 1A

Nazir, A., 3M

Neubrech, Frank, 05

Nguyen, Paul $\vee ., 3 Q$

Nichols, Shane, IW

Nielson, Stew, 3B

Novak, Eric C., $1 \mathrm{~A}$

Nusir, Ahmad I., 3Q

Nyk, M., 1K, 2M

Ocola, Leonidas E., OR

Olesiak-Banska, Joanna, OV

Olivieri, Anthony, 15

Oshikane, Y., 17

Pan, Deng, 00

Panaro, S., 3M

Peckus, Domantas, 2J

Pedersen, K., 08

Pelizzo, Maria G., 2W, $2 Y$

Perera, Chamanei S., 2E

Petranovskii, Vitalii, $2 \mathrm{H}$

Petrova, Rumyana, 3B

Piątkowski, D., 1K, 2M

Pinchuk, Anatoliy O., 14

Proietti Zaccaria, R., 3M

Prymaczek, A., $1 \mathrm{~K}$

Qiu, Ziwei, OR

Rainer, Th., 1M
Rani, Preeti, OW

Reyes, Roberto L., 36

Roberts, Ann, 21

Robinson, Hans D., 36

Roper, D. Keith, 30, 32, 30

Rossouw, David, $2 Z$

Rui, G., 1D

Saastamoinen, Toni, $2 \mathrm{U}$

Sakoda, Kazuaki, OR

Samoc, Marek, OV

Samusev, Anton K., 2R

Santos, Webster L., 36

Sarangan, A. M., 1D

Sarychev, Andrey K., 3C

Savinov, Vassili, OM

Schäferling, Martin, 05

See, Erich M., 36

Shahbazyan, Tigran V., OF

Silvestrini, Simone, $2 \mathrm{~W}$

Simovski, Constantin R., 2R

Sinev, Ivan S., 2R

Sinha, Ravindra Kumar, OW

Skovsen, E., 08

Smolentseva, Elena, $2 \mathrm{H}$

Sǿndergaard, T., 08

Spendier, Kathrin, 14

Stiebing, M., 1M

Strohfeldt, Nikolai, 05

Syrenova, Svetlana, 10

Takahara, Junichi, 2V

Tamulevičiene, Asta, 2J

Tamulevičius, Sigitas, $2 \mathrm{~J}$

Tamulevičius, Tomas, 2J

Tan, Xiaodi, $O Q$

Tanaka, Takuo, 2Q

Tessarolo, Enrico, 2W

Tittl, Andreas, 05

Toma, A., 3M

Toroghi, S., 1B

Tsai, Din Ping, OM

van den Berg, Albert, $2 S$

Vasiliauskas, Andrius, 2J

Vaskin, Alexander V., 3C

Velizhanin, Kirill A., OF

Verbiest, Thierry, 3H

Vernon, Kristy C., 2E

Vo, Thanh Phong, 3L

Voroshilov, Pavel M., 2R

Wadell, Carl, 10

Wang, H., 3M

Wei, Hong, 0O, $\mathrm{OZ}$

Wiedemair, Justyna, 2S

Wu, Pin Chieh, OM

$X U$, Hongxing, $00,0 Z$

Yang, Ying-Ying, 2G

Zasedatelev, Anton, 3E

Zhai, Tianrui, $1 \mathrm{G}$

Zhang, Ling, $2 \mathrm{G}$

Zhang, Xinping, $1 G$

Zheludev, Nikolay I., OM 
Zhou, Wei, OR

Zuccon, Sara, 2W, $2 Y$

Zuppella, Paola, 2W, 2 Y

Proc. of SPIE Vol. $9163916301-9$

Downloaded From: https://www.spiedigitallibrary.org/conference-proceedings-of-spie on 26 Apr 2023 Terms of Use: https://www.spiedigitallibrary.org/terms-of-use 
Proc. of SPIE Vol. $9163916301-10$

Downloaded From: https://www.spiedigitallibrary.org/conference-proceedings-of-spie on 26 Apr 2023 Terms of Use: https://www.spiedigitallibrary.org/terms-of-use 


\section{Conference Committee}

Symposium Chairs

Satoshi Kawata, Osaka University (Japan)

Manijeh Razeghi, Northwestern University (United States)

Symposium Co-chairs

David L. Andrews, University of East Anglia Norwich (United Kingdom) James G. Grote, Air Force Research Laboratory (United States)

Conference Chair

Allan D. Boardman, University of Salford (United Kingdom)

Conference Co-chair

Din Ping Tsai, National Taiwan University (Taiwan)

Conference Program Committee

Martin Aeschlimann, Technische Universität Kaiserslautern (Germany)

Harry A. Atwater Jr., California Institute of Technology (United States)

David J. Bergman, Tel Aviv University (Israel)

Harald W. Giessen, Universität Stuttgart (Germany)

Jean-Jacques Greffet, Institut d'Optique Graduate School (France)

Naomi J. Halas, Rice University (United States)

Martti Kauranen, Tampere University of Technology (Finland)

Satoshi Kawata, Osaka University (Japan)

Fritz Keilmann, LASNIX (Germany)

Dai-Sik Kim, Seoul National University (Korea, Republic of)

Laurens K. Kuipers, FOM Institute for Atomic and Molecular Physics (Netherlands)

Olivier J. F. Martin, École Polytechnique Fédérale de Lausanne

(Switzerland)

Peter Nordlander, Rice University (United States)

Lukas Novotny, University of Rochester (United States)

Vahid Sandoghdar, ETH Zürich (Switzerland)

George C. Schatz, Northwestern University (United States)

Tigran V. Shahbazyan, Jackson State University (United States)

Vladimir M. Shalaev, Purdue University (United States)

Gennady B. Shvets, Institute for Fusion Studies (United States)

Niek F. van Hulst, ICFO - Institut de Ciències Fotòniques (Spain)

Hongxing $\mathbf{X u}$, Institute of Physics (China) 
Nikolay I. Zheludev, University of Southampton (United Kingdom)

Joseph Zyss, École Normale Supérieure de Cachan (France)

\section{Session Chairs}

1 Towards the Future

Din Ping Tsai, National Taiwan University (Taiwan)

2 Active, Gain, Spasing, and Applications

Joel Bellessa, Université Claude Bernard Lyon 1 (France)

3 Nonlinearity, Strings, and Models

Kevin F. MacDonald, University of Southampton (United Kingdom)

4 Graphene Plasmonics

Harry A. Atwater Jr., California Institute of Technology (United States)

5 Spasing, Sensors, and Waveguide

Joel Bellessa, Université Claude Bernard Lyon 1 (France)

6 Meta Outcomes

Ai Qun Liu, Nanyang Technological University (Singapore)

7 Plasmons, Control, and Tunability I

Uriel Levy, The Hebrew University of Jerusalem (Israel)

8 Coupling and Nonlinearity

Ewold Verhagen, FOM Institute for Atomic and Molecular Physics (Netherlands)

9 Nanoscale Activity I

Greg Sun, University of Massachusetts Boston (United States)

10 Nanoscale Activity II

Alexander Benz, Sandia National Laboratories (United States)

11 Plasmons, Control, and Tunability II

Tigran V. Shahbazyan, Jackson State University (United States)

12 On the Nanoscale Again

Laurens K. Kuipers, FOM Institute for Atomic and Molecular

Physics (Netherlands)

13 About Nanoparticles

Christos Tserkezis, Centro de Fisica de Materiales (Spain) 
14 Plasmons, Nanostructures, and Modeling

Otto L. Muskens, University of Southampton (United Kingdom)

15 Plasmons, Control, and Sensing I

Augustine M. Urbas, Air Force Research Laboratory (United States)

16 Plasmons, Control, and Sensing II

Christoph Lienau, Carl von Ossietzky Universität Oldenburg

(Germany)

17 Pathways to Applications I

Siying Peng, California Institute of Technology (United States)

18 Pathways to Applications II

Carl Wadell, Chalmers University of Technology (Sweden) 
Proc. of SPIE Vol. $9163916301-14$

Downloaded From: https://www.spiedigitallibrary.org/conference-proceedings-of-spie on 26 Apr 2023 Terms of Use: https://www.spiedigitallibrary.org/terms-of-use 